\title{
Maximum gap in cyclotomic polynomials
}

\author{
Ala’a Al-Kateeb* Mary Ambrosino ${ }^{\dagger}$ Hoon Hong ${ }^{\ddagger}$ Eunjeong Lee ${ }^{\S}$
}

January 24,2020

\begin{abstract}
Cyclotomic polynomials play fundamental roles in number theory, combinatorics, algebra and their applications. Hence their properties have been extensively investigated. In this paper, we study the maximum gap $g$ (maximum of the differences between any two consecutive exponents). In 2012, it was shown that $g\left(\Phi_{p_{1} p_{2}}\right)=p_{1}-1$ for primes $p_{2}>p_{1}$. In 2017 , based on numerous calculations, the following generalization was conjectured: $g\left(\Phi_{m p}\right)=\varphi(m)$ for square free odd $m$ and prime $p>m$. The main contribution of this paper is a proof of this conjecture.
\end{abstract}

Key Words: Cyclotomic polynomials, inverse cyclotomic polynomials, maximum gap. 2010 Mathematics Subject Classification. 11B83, 11C08.

\section{Introduction}

The $n$-th cyclotomic polynomial $\Phi_{n}$ is defined as the monic polynomial in $\mathbb{Z}[x]$ whose complex roots are the primitive $n$-th roots of unity. The cyclotomic polynomials play fundamental roles in number theory, algebra, combinatorics and their applications, which motivated the extensive investigation on its structure, for instance 'height', 'jump', and 'gap'.

The investigation on height (maximum absolute value of coefficients) was initiated by the finding that the height can be bigger than 1 (as exhibited by $n=105$ ). It has produced numerous results, to list a few: upper bound 11, 10, 6, 12, 36, 17, 32, 7, 9, 13, 31, realizability [37, 29, 18, 19, 23, 21, 22, 35, 26], and flatness [8, 16, 27, 28, 39. The investigation on jumps (a jump happens when two consecutive coefficients are different) was initiated by Bzdęga [14] which was motivated by the work of Gallot and Moree [21]. A sharp bound of the number of jumps for ternary cyclotomic polynomials is provided in [15].

The investigation on maximum gap (maximum difference between the consecutive exponents, see Definition [10) was motivated by its need for analyzing the complexity [25] of a certain cryptographic pairing operation over elliptic curves [20, 30, 40. Later it became a problem on its own because it could be viewed as a first step toward understanding of sparsity structure of cyclotomic polynomials. In 2012 24], it was shown that the maximum gap for binary cyclotomic polynomial $\Phi_{p_{1} p_{2}}$ is $p_{1}-1$, that is, $g\left(\Phi_{p_{1} p_{2}}\right)=p_{1}-1$. In 2014, Moree 34 revisited the result and provided an inspiring conceptual proof by making a connection to numerical semigroups of embedding dimension two. In 2016, Zhang [38] gave a simpler proof, along with the result on the number of occurrences of the maximum gaps. Concurrently, Camburu, et al. [15] communicated an even simpler proof by Kaplan. Thus we feel that the investigation of maximum gap of binary cyclotomic polynomials is practically completed. Hence, the next natural challenge is to study the maximum gap of ternary cyclotomic polynomials, and ultimately, arbitrary cyclotomic polynomials.

In this paper, we tackle the maximum gap in arbitrary case. The following three graphs visualize the relation between $n$ and $g\left(\Phi_{n}\right)$, where the horizontal axis stands for $n$ and the vertical axis for $g\left(\Phi_{n}\right)$.

\footnotetext{
*Department of Mathematics, Yarmouk university, Irbid, Jordan (alaa.kateeb@yu.edu.jo)

${ }^{\dagger}$ Department of Mathematics, North Carolina State University, Raleigh, USA (mary.e.ambrosino@gmail.com)

${ }_{\ddagger}^{\ddagger}$ Department of Mathematics, North Carolina State University, Raleigh, USA (hong@ncsu.edu)

$\S$ Institute of Mathematical Sciences, Ewha Womans University, Seoul, Republic of Korea (ejlee127@gmail.com)
} 
(A) arbitrary $n$

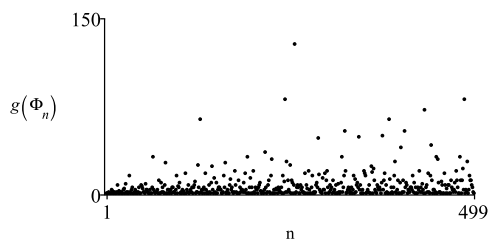

(B) odd square-free $n$

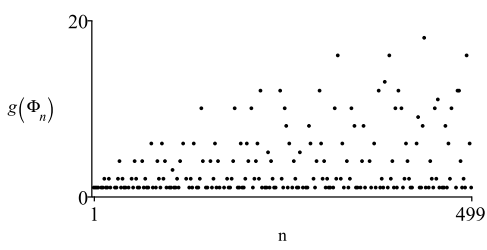

(C) $n=m p$ for $m=105, p>7$ prime

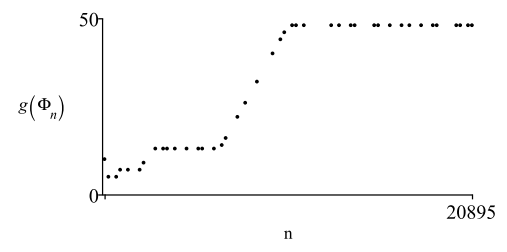

Graph A shows $g\left(\Phi_{n}\right)$ for arbitrary $n$ up to 499. It is not easy to discern any pattern on the relation between $n$ and $g\left(\Phi_{n}\right)$, mainly because there are several very large values of $g\left(\Phi_{n}\right)$, pushing down other values toward 0 . Close inspection shows that those $n$ are usually non-square-free. In fact it can be easily explained by the basic property of cyclotomic polynomial

$$
\Phi_{n}(x)=\Phi_{\operatorname{radical}(n)}\left(x^{\frac{n}{\operatorname{radical}(n)}}\right) \quad \text { which implies } \quad g\left(\Phi_{n}\right)=\frac{n}{\operatorname{radical}(n)} g\left(\Phi_{\operatorname{radical}(n)}\right)
$$

The factor $\frac{n}{\text { radical }(n)}$ contributes to the largeness. Thus we will restrict the study to square-free $n$. But then we recall another basic property

$$
\Phi_{2 n}(x)= \pm \Phi_{n}(-x) \quad \text { in turn } \quad g\left(\Phi_{2 n}\right)=g\left(\Phi_{n}\right) .
$$

Hence we will further restrict the study to odd square-free $n$.

Graph B shows $g\left(\Phi_{n}\right)$ for only odd square-free $n$ up to 499. Now we see some patterns: there are several horizontal lines, that is, several values of $n$ have same values for $g\left(\Phi_{n}\right)$. Close inspection shows that many of them have same prime factors, except the biggest prime factor. This motivates us to restrict $n$ to be $m p$ for a fixed odd square-free $m$ and arbitrary prime $p$ which is bigger than all prime factors of $m$.

Graph C shows $g\left(\Phi_{n}\right)$ for $n$ where $n=m p$ for $m=105$ and arbitrary prime $p$ which is bigger than all prime factors of $m$. Now we finally see some striking patterns. The graph appears to be a piece-wise linear curve with several flat line segments. In particular, there is a flat line apparently forever to the right. Close observation shows that the flat line starts from the smallest prime $p>m$ and the value is $\varphi(m)$. In 2017 [3, 4, through numerous computational confirmation, it was conjectured that the phenomena always hold. In fact, it is consistent with the previous result in [24], that is, for any two primes $p_{1}<p_{2}$, we have $g\left(\Phi_{p_{1} p_{2}}\right)=p_{1}-1=\varphi(m)$, when we let $m=p_{1}$ and $p=p_{2}$. The main contribution of this paper is to prove this conjecture.

Theorem 1 (Main). If $m$ is a square-free odd integer and $p>m$ is a prime, then

$$
g\left(\Phi_{m p}\right)=\varphi(m)
$$

For proving this result, we began by reviewing various proof techniques used for studying cyclotomic polynomials. We noticed that a fruitful technique has been dividing the $\Phi_{m p}$ into several blocks (Notation 11) and investigate and exploit their relations. Several nice relations (such as repetition and invariance) were observed by [5, 27, 28]. See [1, 2] for a list of those relations. Those relations have been successively used for computing cyclotomic polynomials efficiently by Arnold and Monagan [5] and for studying flatness by Kaplan [27, 28.

Encouraged by the effectiveness of the relations, we also applied them to the study of maximum gap. The relations allowed us to focus on a few representative blocks instead of all the blocks of the cyclotomic polynomial. However, the relations were not enough for proving the conjecture, mainly due to the lack of understanding of the representative blocks. In [1, 2, an explicit expression for the blocks was derived. The explicit expression for the blocks allowed us to prove the conjecture for a certain family of cyclotomic polynomials. However, the explicit expression was not enough for proving the full conjecture, mainly because 
it is a sum of several polynomials. The summation could cause introduction and cancellation of terms, resulting in shrinking, enlarging, splitting of gaps, which is very difficult to analyze.

Then, we hit on the key structural observation: All representative blocks are divisible by the $m$-th inverse cyclotomic polynomial (for the definition of inverse cyclotomic polynomial, see [33] and Definition 5). We captured the observation by deriving another expression for blocks, that makes the divisibility explicit (Theorem 22). Using the new explicit expression for the blocks along with the known relations among blocks, we were able to show that the maximum gaps within and between blocks are all less than or equal to $\varphi(m)$, finally proving the conjecture. We hope that the structural result (Theorem 2) could be useful for investigating other properties of cyclotomic polynomials.

The paper is structured as follows: In section 2 we review a usual division of $\Phi_{m p}$ into several blocks (Notation 1) and list several known or easily provable relations among blocks (Lemma 6). Then we prove the key structural result on blocks (Theorem 2). In section 3, using the key structural result for blocks along with the relations among blocks, we show that the maximum gaps within and between blocks are all less than or equal to $\varphi(m)$, finally proving the conjecture.

\section{Blocks}

In this section we review a usual division of $\Phi_{m p}$ into several blocks. Then we study each block and their relations. Lemma 6 list some known or new relations among blocks, explicitly and concisely. Theorem 2 contains the key finding on the structure of each block. It provides an explicit expression, which shows that almost all blocks are divisible by an inverse cyclotomic polynomial.

Notation 1 (Division and blocks). We divide $\Phi_{m p}$ as follows:

$$
\begin{aligned}
\Phi_{m p} & =\sum_{i=0}^{\varphi(m)-1} f_{m, p, i} x^{i p} & \text { where } \operatorname{deg} f_{m, p, i}<p \\
f_{m, p, i} & =\sum_{j=0}^{q} f_{m, p, i, j} x^{j m} & \text { where } \operatorname{deg} f_{m, p, i, j}<m
\end{aligned}
$$

where $q=$ quo $(p, m)$ and $r=\operatorname{rem}(p, m)$. Observing the sizes of each blocks, we call

1. $f_{m, p, i}$ a $p$-block

2. $f_{m, p, i, j}$ an $m$-block for $j<q$

3. $f_{m, p, i, q}$ an $r$-block

Example 2. Let $m=15$ and $p=53$. Then $\varphi(m)=8, q=3$ and $r=8$. The following diagram illustrates the division of $\Phi_{m p}$ into $p$-blocks and then the division of each p-block into three $m$-blocks and one r-block.

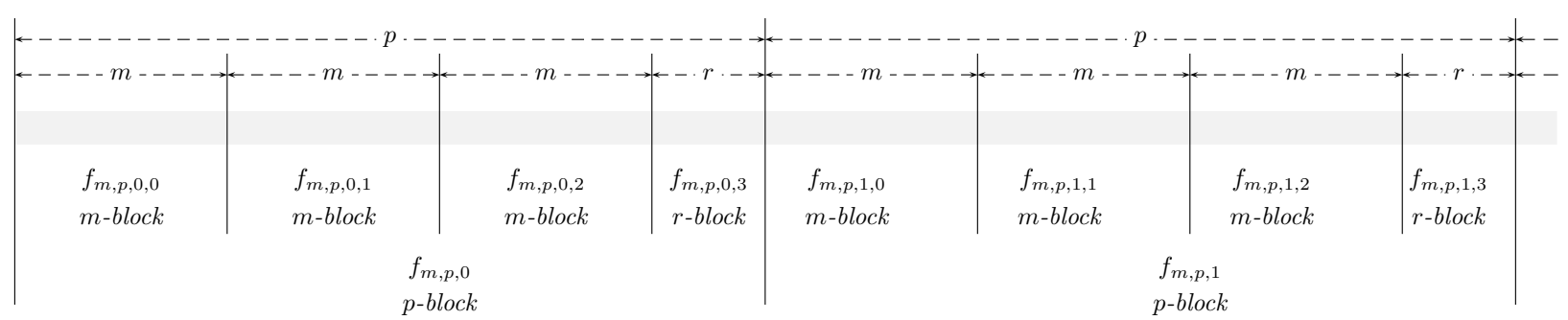

where the long shaded strip stand for the terms in $\Phi_{m p}$ (increasing order in the exponents). In order to avoid going off the right margin, we cut the diagram off at the margin. 
Remark 3. In Notation 1, the upper bound $\varphi(m)-1$ for the index $i$ is from the following observation:

$$
\text { quo }\left(\operatorname{deg} \Phi_{m p}, p\right)=\operatorname{quo}(\varphi(m p), p)=\operatorname{quo}(\varphi(m)(p-1), p)=\varphi(m)-1
$$

Now we "conquer" the blocks. We begin by finding relation between blocks (Lemma 6). Then, using the relation, we derive an explicit expression for blocks (Theorem 21). This is the "key" result, since it will play a crucial role in the remainder of the paper. We end this subsection, by deriving a few immediate consequences (Corollaries 9 and 8). To present all these results compactly, we will introduce a few notations and notions.

Notation 4 (Block operations). Let $h=\sum_{k=0}^{m-1} h_{k} x^{k} \cong\left(h_{0}, \ldots, h_{m-1}\right)$. Let $0 \leq s<m$. Then

truncate : $\mathcal{T}_{s} h \quad=\operatorname{rem}\left(h, x^{s}\right) \quad \cong\left(h_{0}, \ldots, h_{s-1}\right)$

rotate $: \mathcal{R}_{m, s} h=\operatorname{rem}\left(x^{m-s} h, x^{m}-1\right) \cong\left(h_{s}, h_{s+1}, \ldots, h_{m-1}, h_{0}, \ldots, h_{s-1}\right)$

Definition 5 (Inverse cyclotomic polynomial). The $m$-th inverse cyclotomic polynomial $\Psi_{m}$ is defined as

$$
\Psi_{m}=\frac{x^{m}-1}{\Phi_{m}}
$$

We will use the notation $\psi(m)=\operatorname{deg} \Psi_{m}$. See [33] for various interesting properties.

From now on, through out the paper, we will assume that $p>m$ and $0 \leq i \leq \varphi(m)-1$.

Lemma 6 (Relation between blocks). We have

1. $f_{m, p, i, 0}=f_{m, p, i, j} \quad$ for $0 \leq j \leq q-1$

2. $f_{m, p, i, q}=\mathcal{T}_{r} f_{m, p, i, 0}$

3. $f_{m, p, i+1,0}=\mathcal{R}_{m, r} f_{m, p, i, 0}-a_{i+1} \Psi_{m}$

Remark 7. The above lemma shows that the blocks $f_{m, p, i, 0}$ play role of "representatives".

Proof. We begin by representing $\Phi_{m p}$ as a formal power series, as usual:

$$
\Phi_{m p}=\frac{\Phi_{m}\left(x^{p}\right)}{\Phi_{m}(x)}=\Phi_{m}\left(x^{p}\right) \frac{1}{x^{m}-1} \Psi_{m}=-\sum_{s \geq 0} a_{s} x^{s p} \sum_{u \geq 0} x^{u m} \Psi_{m}=\sum_{s \geq 0} x^{s p} \sum_{u \geq 0} x^{u m} C_{s}
$$

where $C_{s}=-a_{s} \Psi_{m}$. Thus $\Phi_{m p}$ is the $p$-shifted sum of the $m$-shifted sum of $C_{s}$. It is illustrated by the first diagram in Figure 1. Now we are ready to prove each claim one by one. For this we will repeatedly refer to the second diagram in Figure 1 Note that the $m$-block $f_{m, p, i, 0}$ can be obtained from $\Phi_{m p}$ by taking the slice of length $m$ from $i p$. It is illustrated by the first block.

1. $f_{m, p, i, 0}=f_{m, p, i, j}$ for $0 \leq j \leq q-1$

The $m$-block $f_{m, p, i, j}$ is obtained by taking the slice of length $m$ from $i p+j m$. It is illustrated by the second block. Comparing the two blocks $f_{m, p, i, 0}$ and $f_{m, p, i, j}$, we observe that $f_{m, p, i, 0}=f_{m, p, i, j}$, that ism, $f_{m, p, i, j}$ does not depend on $j$.

2. $f_{m, p, i, q}=\mathcal{T}_{r} f_{m, p, i, 0}$

The $r$-block $f_{m, p, i, q}$ is obtained by taking the slice of length $r$ from $i p+q m$. It is illustrated by the third block. Comparing the two blocks $f_{m, p, i, 0}$ and $f_{m, p, i, q}$, we observe that $f_{m, p, i, q}$ consists of the first $r$ terms of $f_{m, p, i, 0}$, that is, $f_{m, p, i, q}=\mathcal{T}_{r} f_{m, p, i, 0}$.

3. $f_{m, p, i+1,0}=\mathcal{R}_{m, r} f_{m, p, i, 0}-a_{i+1} \Psi_{m}$

The $m$-block $f_{m, p,(i+1), 0}$ is obtained by taking the slice of length $m$ from $(i+1) p$. It is illustrated by the fourth block. Comparing the two blocks $f_{m, p, i, 0}$ and $f_{m, p, i+1,0}$, we observe the followings. 


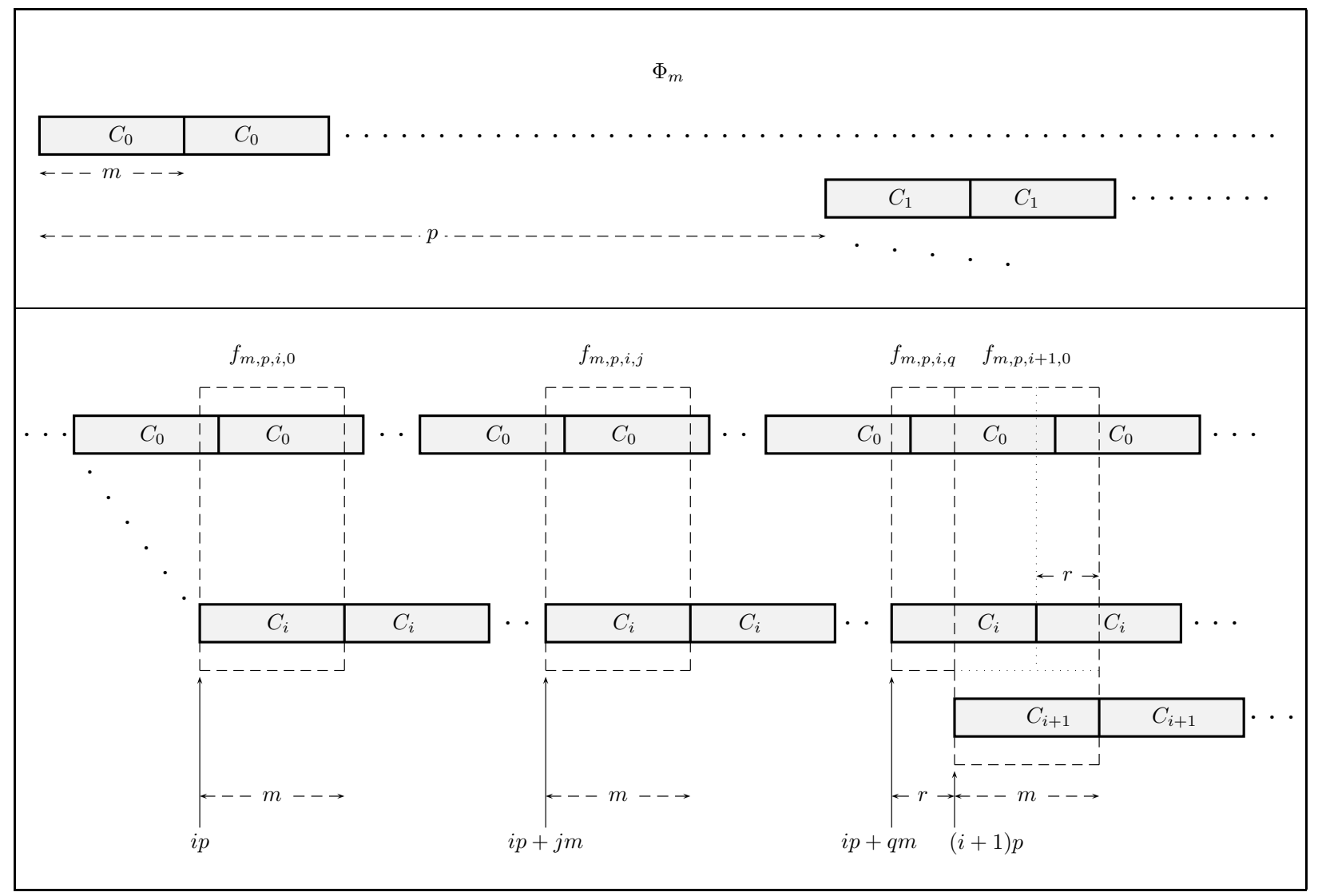

Figure 1: Relation between blocks

(a) The $m$-block $f_{m, p, i, 0}$ consists of $i+1$ rows and the $m$-block $f_{m, p, i+1,0}$ consists of $i+2$ rows.

(b) The sum of the first $i+1$ rows of $f_{m, p, i+1,0}$ is the rotation of $f_{m, p, i, 0}$ by $r$ modulo $m$. This happens because $\operatorname{rem}(p, m)=r$.

(c) The last row of $f_{m, p, i+1,0}$ is $C_{i+1}$

Putting together, we have $f_{m, p, i+1,0}=\mathcal{R}_{m, r} f_{m, p, i, 0}+C_{i+1}=\mathcal{R}_{m, r} f_{m, p, i, 0}-a_{i+1} \Psi_{m}$.

Theorem 2 (Explicit expression for blocks). We have

$$
f_{m, p, i, 0}=\Theta_{i} \Psi_{m}
$$

where $\Theta_{i}$ is defined by

$$
\Theta_{i}=\operatorname{rem}\left(w_{i}\left(x^{m-r}\right), \Phi_{m}\right) \quad w_{i}(x)=-\sum_{0 \leq s \leq i} a_{s} x^{i-s} \quad \Phi_{m}=\sum_{s} a_{s} x^{s}
$$

Proof. We prove the claim by induction on $i$. When $i=0$. The claim is immediate from

$$
\begin{aligned}
f_{m, p, 0, j} & =-a_{0} \Psi_{m} \\
\Theta_{0} \Psi_{m} & =\operatorname{rem}\left(w_{0}\left(x^{m-r}\right), \Psi_{m}\right) \Psi_{m}=-a_{0} \Psi_{m}
\end{aligned}
$$


Assume that the claim is true up to $i-1$. It suffices to show the claim holds for $i$. Note

$$
\begin{array}{rlrl}
f_{m, p, i, j} & =\mathcal{R}_{m, r} f_{m, p, i-1, j}-a_{i} \Psi_{m} & & \text { from Lemma 6+3 } \\
& =\operatorname{rem}\left(x^{m-r} f_{m, p, i-1, j}, x^{m}-1\right)-a_{i} \Psi_{m} & & \text { from Notation } 4 \\
& =\operatorname{rem}\left(x^{m-r} \Theta_{i-1} \Psi_{m}, x^{m}-1\right)-a_{i} \Psi_{m} & & \text { from the induction hypothesis } \\
& =\operatorname{rem}\left(x^{m-r} \Theta_{i-1} \Psi_{m}, \Phi_{m} \Psi_{m}\right)-a_{i} \Psi_{m} & & \text { since } x^{m}-1=\Phi_{m} \Psi_{m} \\
& =\left(\operatorname{rem}\left(x^{m-r} \Theta_{i-1}, \Phi_{m}\right)-a_{i}\right) \Psi_{m} & & \text { by factoring out } \Psi_{m} \\
& =\operatorname{rem}\left(x^{m-r} \Theta_{i-1}-a_{i}, \Phi_{m}\right) \Psi_{m} & & \text { since } \operatorname{deg} \Phi_{m}>\operatorname{deg} a_{i} \\
& =\operatorname{rem}\left(-x^{m-r} \sum_{s=0}^{i-1} a_{s} x^{(m-r)(i-1-s)}-a_{i}, \Phi_{m}\right) \Psi_{m} & & \text { from the definition of } \Theta_{i-1} \\
& =\operatorname{rem}\left(-\sum_{s=0}^{i-1} a_{s} x^{(m-r)(i-s)}-a_{i}, \Phi_{m}\right) \Psi_{m} & & \text { by pushing } x^{m-r} \text { into the summation } \\
& =\operatorname{rem}\left(-\sum_{s=0}^{i} a_{s} x^{(m-r)(i-s)}, \Phi_{m}\right) \Psi_{m} & & \\
& =\Theta_{i} \Psi_{m} & & \text { since } a_{i}=a_{i} x^{(m-r)(i-i)} \\
& \text { from the definition of } \Theta_{i}
\end{array}
$$

as desired

From the explicit expression of $m$-blocks, we can extract two immediate but useful properties of $m$-blocks.

Corollary 8 (Non-zero). $f_{m, p, i, 0} \neq 0$

Proof. Suppose that $f_{m, p, i, 0}=0$ for some $i$. Since $\Psi_{m} \neq 0$, from Theorem 2, we have

$$
\Phi_{m}(x) \mid w_{i}\left(x^{m-r}\right)
$$

Let $\zeta$ be an $m$-th primitive root of unity. Then we have $w_{i}\left(\zeta^{m-r}\right)=0$, because $m$ and $m-r$ are relatively prime. Note that $\zeta^{m-r}$ is also an $m$-th primitive root of unity and the degree of its minimal polynomial is $\varphi(m)$. However, $w_{i}(x)=a_{0} x^{i}+\cdots \neq 0$ and $\operatorname{deg} w_{i}(x)<\varphi(m)$. Contradiction.

Corollary 9 (Degree difference). $\operatorname{deg} f_{m, p, i, 0}-\operatorname{tdeg} f_{m, p, i, 0} \geq \psi(m)$ and the equality holds when $i=0$.

Proof. Note

$$
\begin{array}{rlrl} 
& \operatorname{deg} f_{m, p, i, 0}-\operatorname{tdeg} f_{m, p, i, 0} & \\
= & \operatorname{deg}\left(\Theta_{i} \Psi_{m}\right)-\operatorname{tdeg}\left(\Theta_{i} \Psi_{m}\right) & & \text { from Theorem 2 } \\
= & \left(\operatorname{deg} \Theta_{i}+\operatorname{deg} \Psi_{m}\right)-\left(\operatorname{tdeg} \Theta_{i}+\operatorname{tdeg} \Psi_{m}\right) & & \text { by expanding } \\
= & \operatorname{deg} \Theta_{i}+\psi(m)-\left(\operatorname{tdeg} \Theta_{i}+0\right) & & \text { from } \operatorname{tdeg} \Psi_{m}=0 \\
= & \operatorname{deg} \Theta_{i}-\operatorname{tdeg} \Theta_{i}+\psi(m) & & \text { by simplifying }
\end{array}
$$

Hence

$$
\begin{aligned}
& \operatorname{deg} f_{m, p, i, 0}-\operatorname{tdeg} f_{m, p, i, 0} \geq \psi(m) \quad \text { since } \operatorname{tdeg} \Theta_{i} \leq \operatorname{deg} \Theta_{i} \\
& \operatorname{deg} f_{m, p, i, 0}-\operatorname{tdeg} f_{m, p, i, 0}=\psi(m) \quad \text { since } \Theta_{0}=-1
\end{aligned}
$$




\section{Gaps}

In this section, we will prove the main result of this paper (Theorem 1) utilizing the block structures found in the previous section (Section 22). For this, let us first recall the precise definition of maximum gap [24].

Definition 10 (Maximum gap). Let $f=a_{1} x^{e_{1}}+a_{2} x^{e_{2}}+\cdots+a_{n} x^{e_{n}}$, where $a_{1}, \cdots, a_{n} \neq 0$ and $e_{1}<\cdots<e_{n}$. Then the maximum gap of $f$, written as $g(f)$ is given by

$$
g(f)=\max _{0 \leq i<n} e_{i+1}-e_{i}
$$

Lemma 6-1, Theorem 2 and Corollary 8 motivate the following notations of the gaps within and between the blocks in $\Phi_{m p}$.

Notation 11 (Block gaps). Let

$$
\begin{aligned}
& g_{m, i}^{\mathrm{w}}: \text { maximum gap within } f_{m, p, i, 0} \quad \text { (the first } m \text {-block in } i \text {-th } p \text {-block) } \\
& g_{r, i}^{\mathrm{w}} \quad: \text { maximum gap within } f_{m, p, i, q} \quad \text { (the } r \text {-block in } i \text {-th } p \text {-block) } \\
& g_{m, i}^{\mathrm{b}} \quad: \quad \text { gap between } f_{m, p, i, 0} \text { and } f_{m, p, i, 1} \quad \text { (the first two } m \text {-blocks within the } i \text {-th } p \text {-block) } \\
& g_{r, i}^{\mathrm{b}} \quad: \quad \text { gap between } f_{m, p, i, q-1} \text { and } f_{m, p, i, q} \quad \text { (the last } m \text {-block and the } r \text {-block within the } i \text {-th } p \text {-block) } \\
& g_{p, i}^{\mathrm{b}} \quad: \quad \text { gap between } f_{m, p, i-1} \text { and } f_{m, p, i} \quad \text { (the } i \text {-th } p \text {-block and the }(i-1) \text {-th } p \text {-block) }
\end{aligned}
$$

In some cases, the above quantities are meaningless. In such cases, for the sake of notational simplicity, we will assign 0 to them, that is,

$$
\begin{array}{lll}
g_{r, i}^{\mathrm{w}}=0 & \text { if } & f_{m, p, i, q}=0 \\
g_{m, i}^{\mathrm{b}}=0 & \text { if } & q=1 \\
g_{r, i}^{\mathrm{b}}=0 & \text { if } & f_{m, p, i, q}=0 \\
g_{p, i}^{\mathrm{b}}=0 & \text { if } & i=0
\end{array}
$$

Example 12. We illustrate the notations for $m=15$ and $p=53$ as in Example 0 . The following diagram illustrates the division of $\Phi_{m p}$ from the $p$-block when $i=2$ to the beginning of the p-block when $i=4$. The dark bar stands for non-zero term and the white bar stands for zero term.

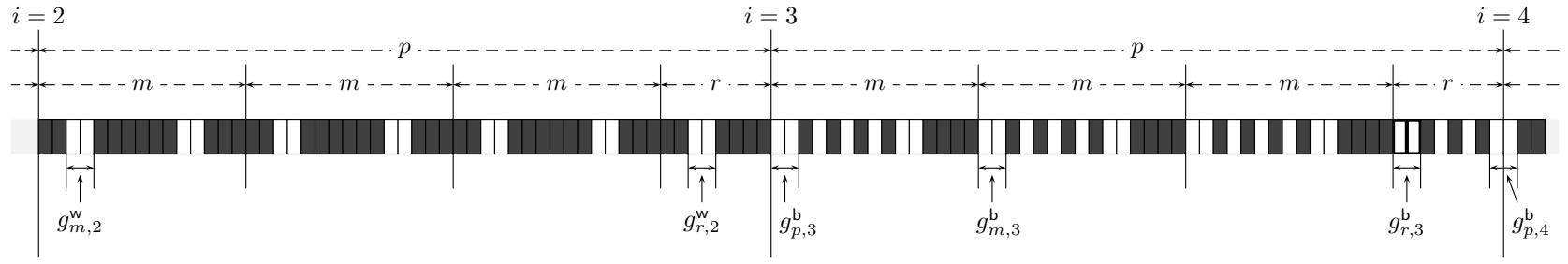

Next we express the maximum gap of $\Phi_{m p}$ in terms of the block gaps.

Lemma 13. $g\left(\Phi_{m p}\right)=\max _{0 \leq i \leq \varphi(m)-1}\left\{g_{m, i}^{\mathrm{w}}, \quad g_{r, i}^{\mathrm{w}}, \quad g_{m, i}^{\mathrm{b}}, \quad g_{r, i}^{\mathrm{b}}, \quad g_{p, i}^{\mathrm{b}}\right\}$

Proof. Immediate from Lemma 6 1, Theorem 2 and Corollary 8

The above lemma tells us that we need to investigate the block gaps: $g_{m, i}^{\mathrm{w}}, g_{r, i}^{\mathrm{w}}, g_{m, i}^{\mathrm{b}}, g_{r, i}^{\mathrm{b}}, g_{p, i}^{\mathrm{b}}$. In particular, we need to show that each block gap is less than or equal to $\varphi(m)$ and at least one of them is equal to $\varphi(m)$. In the following, we will do so one by one.

Lemma 14. $g_{m, i}^{\mathrm{w}} \leq \varphi(m)$

Proof. We will first prove a more general claim. Then the lemma will follow immediately. 
1. Let $h=v \Psi_{m}$ such that $\operatorname{deg} h<m$. Then $g(h) \leq \varphi(m)$.

We prove the claim by contradiction.

(a) Assume $g(h)>\varphi(m)$. Let

$$
h=\sum_{i=1}^{t} h_{i} x^{e_{i}}
$$

where $0 \leq e_{1}<e_{2}<\cdots<e_{t}<m$ and $h_{1}, \ldots, h_{t} \neq 0$. Then for some $k$ we have

$$
g(h)=e_{k}-e_{k-1}>\varphi(m) .
$$

(b) Note

$$
h \Phi_{m}=\sum_{i=1}^{t} h_{i} x^{e_{i}} \Phi_{m}(x)=\sum_{i=1}^{t} B_{i} \quad \text { where } B_{i}=h_{i} x^{e_{i}} \Phi_{m}(x)
$$

The polynomials $B_{1}, \ldots, B_{t}$ are visualized in the following diagram

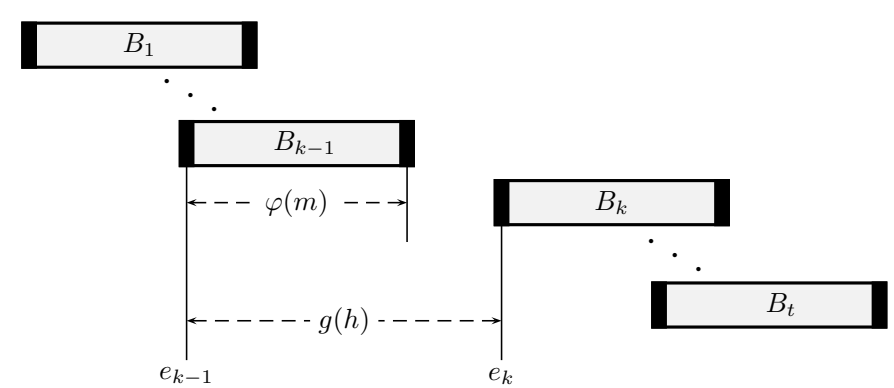

where the black color indicates that the corresponding exponent (term) occurs in the polynomial and the grey color indicates that we do not need to know whether it occurs or not.

Observe that the term $x^{e_{k}}$ appears only in $B_{k}$ and thus it does not get cancelled during the summation. Hence

$$
\operatorname{coeff}_{e_{k}}\left(h \Phi_{m}\right) \neq 0
$$

(c) Note

$$
h \Phi_{m}=v \Psi_{m} \Phi_{m}=v\left(x^{m}-1\right)=v x^{m}-v
$$

Observe

$$
\operatorname{deg} v<\varphi(m)<e_{k}<m \leq \operatorname{tdeg}\left(v x^{m}\right)
$$

Hence

$$
\operatorname{coeff}_{e_{k}}\left(h \Phi_{m}\right)=0
$$

(d) Contradiction.

2. $g_{m, i}^{\mathrm{w}} \leq \varphi(m)$.

Immediate from applying the above claim to the expression in Theorem 2 .

Lemma 15. $g_{r, i}^{\mathrm{w}} \leq \varphi(m)$. 
Proof. When $f_{m, p, i, q}=0$, the claim is vacuously true. When $f_{m, p, i, q} \neq 0$, the claim is immediate from

$$
\begin{aligned}
g_{r, i}^{\mathrm{w}} & =g\left(f_{m, p, i, q}\right) \\
& =g\left(\mathcal{T}_{r} f_{m, p, i, 0}\right) \\
& \leq g\left(f_{m, p, i, 0}\right) \\
& \leq \varphi(m)
\end{aligned}
$$

Lemma 16. $g_{m, i}^{\mathrm{b}} \leq \varphi(m)$

Proof. When $q=1$, the claim is vacuously true. When $q>1$, the claim is immediate from

$$
\begin{aligned}
g_{m, i}^{\mathrm{b}} & =m+\operatorname{tdeg} f_{m, p, i, 1}-\operatorname{deg} f_{m, p, i, 0} \\
& =m+\operatorname{tdeg} f_{m, p, i, 0}-\operatorname{deg} f_{m, p, i, 0} \\
& \leq \varphi(m)
\end{aligned}
$$

from Lemma 6 - 1

from Corollary 9

Lemma 17. $g_{r, i}^{\mathrm{b}} \leq \varphi(m)$ and the equality holds when $i=0$.

Proof. When $f_{m, p, i, q}=0$, the claim is vacuously true. When $f_{m, p, i, q} \neq 0$, note

$$
\begin{aligned}
g_{r, i}^{\mathrm{b}} & =m+\operatorname{tdeg} f_{m, p, i, q}-\operatorname{deg} f_{m, p, i, q-1} & & \\
& =m+\operatorname{tdeg} \mathcal{T}_{r} f_{m, p, i, 0}-\operatorname{deg} f_{m, p, i, 0} & & \text { from Lemma } 6-1,2 \\
& =m+\operatorname{tdeg} f_{m, p, i, 0}-\operatorname{deg} f_{m, p, i, 0} & & \text { since } f_{m, p, i, q} \neq 0 \\
& \leq \varphi(m) & & \text { from Corollary } 9
\end{aligned}
$$

Lemma 18. $g_{p, i}^{\mathrm{b}} \leq \varphi(m)$

Proof. When $i=0$, the claim is vacuously true. Hence from now on we assume that $i \geq 1$. The proof is a bit long. Hence we will divide the proof into several claims and their proofs.

1. $\operatorname{deg} f_{m, p, i, 0}=\operatorname{deg} \mathcal{R}_{m, r} f_{m, p, i-1,0}$

(a) $\Psi_{m} \mid f_{m, p, i, 0}$ from Theorem 2 ,

(b) $\Psi_{m} \mid \mathcal{R}_{m, r} f_{m, p, i-1,0}$ from Lemma 6 -3 and the step above.

(c) $\mathcal{R}_{m, r} f_{m, p, i-1,0} \neq 0$ from Corollary 8 and Notation 4 ,

(d) $\psi(m) \leq \operatorname{deg} \mathcal{R}_{m, r} f_{m, p, i-1,0}$ from the two steps above.

(e) $\psi(m) \leq \operatorname{deg} f_{m, p, i, 0}$ from Corollary 9 .

(f) $\operatorname{deg} f_{m, p, i, 0}=\operatorname{deg} \mathcal{R}_{m, r} f_{m, p, i-1,0}$ from Lemma 6 -3.

2. $\operatorname{deg} f_{m, p, i-1}=\operatorname{deg} f_{m, p, i, 0}+p-m$

Note

$$
\begin{aligned}
& \operatorname{deg} f_{m, p, i-1} \\
& =\left\{\begin{array}{ll}
\operatorname{deg} f_{m, p, i-1, q}+q m & \text { if } f_{m, p, i-1, q} \neq 0 \\
\operatorname{deg} f_{m, p, i-1,0}+(q-1) m & \text { if } f_{m, p, i-1, q}=0
\end{array} \quad \text { from Notation 1, Lemma 66-1 and Corollary } 8\right. \\
& =\left\{\begin{array}{ll}
\operatorname{deg} \mathcal{R}_{m, r} f_{m, p, i-1,0}-(m-r)+q m & \text { if } f_{m, p, i-1, q} \neq 0 \\
\operatorname{deg} \mathcal{R}_{m, r} f_{m, p, i-1,0}+r+(q-1) m & \text { if } f_{m, p, i-1, q}=0
\end{array} \quad \text { from Notation } 4 \text { and Lemma } 6\right\} 2 \\
& =\operatorname{deg} \mathcal{R}_{m, r} f_{m, p, i-1,0}+p-m \quad \text { from } p=q m+r \\
& =\operatorname{deg} f_{m, p, i, 0}+p-m \quad \text { from the last claim }
\end{aligned}
$$


3. $g_{p, i}^{\mathrm{b}} \leq \varphi(m)$

Note

$$
\begin{aligned}
g_{p, i}^{\mathrm{b}} & =p+\operatorname{tdeg} f_{m, p, i}-\operatorname{deg} f_{m, p, i-1} & & \\
& =p+\operatorname{tdeg} f_{m, p, i, 0}-\left(\operatorname{deg} f_{m, p, i, 0}+p-m\right) & & \text { from the last claim } \\
& =m+\operatorname{tdeg} f_{m, p, i, 0}-\operatorname{deg} f_{m, p, i, 0} & & \text { by simplifying } \\
& \leq \varphi(m) & & \text { from Corollary } 9
\end{aligned}
$$

Finally we "combine" the previous results to prove the main result.

Proof of Main Result (Theorem 1). Recall Lemma 13

$$
g\left(\Phi_{m p}\right)=\max _{0 \leq i \leq \varphi(m)-1}\left\{g_{m, i}^{\mathrm{w}}, \quad g_{r, i}^{\mathrm{w}}, \quad g_{m, i}^{\mathrm{b}}, \quad g_{r, i}^{\mathrm{b}}, \quad g_{p, i}^{\mathrm{b}}\right\}
$$

By applying Lemmas 14, 15, 16, 17 and 18 to the above expression, we finally have

$$
g\left(\Phi_{m p}\right)=\varphi(m)
$$

\section{References}

[1] A. Al-Kateeb. Structures and properties of cyclotomic polynomials. PhD thesis, North Carolina state university, 2016.

[2] A. Al-Kateeb, H. Hong, and E. Lee. Block structure of cyclotomic polynomials. arXiv:1704.04051v2, 2017.

[3] M. Ambrosino. Maximum gap of (inverse) cyclotomic polynomials. PhD thesis, North Carolina state university, 2017.

[4] M. Ambrosino, H. Hong, and E. Lee. Lower bounds for maximum gap in (inverse) cyclotomic polynomials. arxiv.org/abs/1702.07650, 2017.

[5] A. Arnold and M. Monagan. A high-performance algorithm for calculating cyclotomic polynomials. Proceedings of PASCO, ACM Press, pages 112-120, 2010.

[6] G. Bachman. On the coefficients of ternary cyclotomic polynomials. J. Number Theory, 100:104-116, 2003.

[7] G. Bachman. Ternary cyclotomic polynomials with an optimally large set of coefficents. Proc. Amer. Math. Soc, 132(7):1943-1950, 2004.

[8] G. Bachman. Flat cyclotomic polynomials of order three. Bull. London Math. Soc, 38:53-60, 2006.

[9] P. T. Bateman, C. Pomerance, and R. C. Vaughan. On the size of the coefficients of the cyclotomic polynomial. Topics in classical number theory, 1:171-202, 1984.

[10] M. Beiter. Magnitude of the coefficients of the cyclotomic polynomial $F_{p q r}$. Amer. Math. Monthly, $75(4): 370-372,1968$.

[11] D. Bloom. On the coefficients of the cyclotomic polynomials. Amer. Math. Monthly, 75(4):372-377, 1968.

[12] B. Bzdȩga. Bounds on ternary cyclotomic coefficients. Acta Arithmetica, 144(1):5-16, 2010.

[13] B. Bzdęga. On the height of cyclotomic polynomials. Acta Arithmetica, 152(4):349-359, 2012. 
[14] B. Bzdȩga. Jumps of ternary cyclotomic polynomials. Acta Arithmetica, 163(3):203-213, 2014.

[15] O. Camburu, E. Ciolan, P. Moree F. Luca, and I. E. Shparlinski. Cyclotomic coefficients: gaps and jumps. J. Number Theory, 163:211-237, 2016.

[16] S. Elder. Flat cyclotomic polynomials: A new approach. arXiv:1207.5811v1 [math.NT], 2012.

[17] P. Erdős and R. C. Vaughan. Bounds for the $r$-th coefficients of cyclotomic polynomials. J. London Math. Soc, 2:393-400, 1974.

[18] J. Fintzen. Cyclotomic polynomial coefficients $a(n, k)$ with $n$ and $k$ in prescribed residue classes. $J$. Number Theory, 131(10):1852-1863, 2011.

[19] É. Fouvry. On binary cyclotomic polynomials. Algebra and number theory, 7(5):1207-1223, 2013.

[20] D. Freeman, M. Scott, and E. Teske. A taxonomy of pairing-friendly elliptic curves. J. Cryptology, 23(2):224-280, 2009.

[21] Y. Gallot and P. Moree. Neighboring ternary cyclotomic polynomials coefficients differ by at most one. J. Ramanujan Math Soc, 24:235-248, 2009.

[22] Y. Gallot, P. Moree, and H. Hommersom. Value distribution of coefficients of cyclotomic polynomials. Unif. Distrib. Theory, 6:177-206, 2011.

[23] Y. Gallot, P. Moree, and R. Wilms. The family of ternary cyclotomic polynomials with one free prime. Involve, 4(4):317-341, 2011.

[24] H. Hong, E. Lee, H-S. Lee, and C-M. Park. Maximum gap in (inverse) cyclotomic polynomial. J. Number Theory, 132(10):2297-2317, 2012.

[25] H. Hong, E. Lee, H-S. Lee, and C-M. Park. Simple and exact formula for minimum loop length in Ate ${ }_{i}$ pairing based on Brezing-Weng curves. Designs, codes and cryptography, 67(2):271-292, 2013.

[26] C. Ji, W. Li, and P. Moree. Values of coefficients of cyclotomic polynomials II. Discrete Math, 309(6):1720-1723, 2009.

[27] N. Kaplan. Flat cyclotomic polynomials of order three. J. Number Theory, 127(1):118-126, 2007.

[28] N. Kaplan. Flat cyclotomic polynomials of order four and higher. Integers, 10:357-363, 2010.

[29] A. Kosyak, P. Moree, E. Sofos, and B. Zhang. Cyclotomic polynomials with prescribed height. arXiv:1910.01039, 2019.

[30] E. Lee, H-S. Lee, and C-M. Park. Efficient and generalized pairing computation on abelian varieties. IEEE Transactions on Information Theory, 55(4):1793-1803, 2009.

[31] E. Lehmer. On the magnitude of the coefficients of the cyclotomic polynomials. Bull. Amer. Math. Soc, 42:389-392, 1936.

[32] H. L. Montgomery and R. C. Vaughan. The order of magnitude of the $m$-th coefficients of cyclotomic polynomials. Glasgow Math, 27:143-159, 1985.

[33] P. Moree. Inverse cyclotomic polynomials. J. Number Theory, 129(3):667-680, 2009.

[34] P. Moree. Numerical semigroups, cyclotomic polynomials, and Bernoulli numbers. Amer. Math. Monthly, 121(10):890-902, 2014.

[35] P. Moree and E. Roşu. Non-Beiter ternary cyclotomic polynomials with an optimally large set of coefficients. Int. J. Number Theory, 8(8):1883-1902, 2012.

[36] R. C. Vaughan. Bounds for the coefficients of cyclotomic polynomials. The Michigan Mathematical Journal, 21(4):289-295, 1975.

[37] R. C. Vaughan. Coefficients of cyclotomic polynomials and related topics. In Proceedings of the Congress on Number Theory, pages 43-68. Servicio Editorial de la Universidad del Pais Vasco, 1990. 
[38] B. Zhang. Remarks on the maximum gap in binary cyclotomic polynomials. Bull. Math. Soc. Sci. Math. Roumanie (N.S.), 59:109-115, 2016.

[39] B. Zhang. Remarks on the flatness of ternary cyclotomic. Int. J. Number theory, 13(2):529-547, 2017.

[40] C. Zhao, F. Zhang, and J. Huang. A note on the Ate pairing. Int .J. Inf. Secur, 7(6):379-382, 2008. 